\title{
Experiencia de 6 años en la evaluación de las cardiopatías congénitas con resonancia magnética.
}

\author{
Rodrigo Parra R, Lina Cadavid A, Cristian García B, Florencia de Barbieri. \\ Hospital Clínico Pontifica Universidad Católica. Departamento de Radiología, Unidad de Radiología \\ pediátrica.
}

Recibido el 16 de marzo de 2009. Aceptado el 15 de julio de 2009

\section{Resumen}

Rev Chil Cardiol 2009; 28: 165-173

Introducción: La Resonancia Magnética (RM) es de gran ayuda como método complementario en la evaluación de las cardiopatías congénitas debido a que provee información anatómica y funcional.

Objetivo: Describir la utilidad, indicaciones y hallazgos más comunes en la evaluación de las cardiopatías congénitas por RM

Método: Se realizó un análisis retrospectivo de las RM cardíacas efectuadas entre el año 2002 hasta el 2008 en el Hospital Clínico de la Pontificia Universidad Católica de Chile. Se incluyó la totalidad de los pacientes en edad pediátrica y aquellos adultos en los cuales la indicación del examen fue por cardiopatía congénita, tanto para evaluación preoperatoria como para control postquirúrgico. Se realizó estudio anatómico, funcional y se utilizó contraste paramagnético intravenoso en los casos necesarios.

Resultados: Se realizaron 180 estudios. La indicación más frecuente fue la evaluación postoperatoria de la tetralogía de Fallot, seguida por drenaje venoso pulmonar anómalo, miocardiopatía no compactada y coartación de Aorta, entre otros.

Conclusiones: La RM cardíaca es de gran utilidad en la evaluación de las cardiopatías congénitas, ya que demuestra detalles anatómicos con excelente resolución de contraste y provee además información funcional y de dinámicas de flujo.

Palabras Claves: Cardiopatías Congénitas, Resonancia Magnética.

\section{Evaluation of Congenital Heart Disease using Magnetic Resonance Imaging. A 6 year experience}

Background: Magnetic resonance imaging (MRI) is helpful in the evaluation of congenital heart diseases because it provides anatomical and functional information.

Aim: To describe the utility, indications and more commons findings in the evaluation of the congenital heart diseases using MRI.

Method: A retrospective analysis of the cardiac MRI studies in patients with congenital heart diseases between 2002 and 2008 was completed. Children and adults were included. Preoperative evaluation and or post surgical findings were analyzed. Anatomical and functional studies were made and intravenous paramagnetic contrast agents were used in the selected cases.

Results: $180 \mathrm{MRI}$ studies were analyzed. The most frequent indication was corrected Fallot's Tetralogy followed by anomalous pulmonary venous connection, left ventricular non compaction and coarctation of the aorta. Flow dynamics studies were successfully performed in several cases.

Conclusion: Cardiac MRI demonstrates anatomical details with excellent contrast resolution and allows functional and flow dynamics studies.

Key Words: Congenital Heart Diseases, Magnetic Resonance Imaging

Correspondencia: Rodrigo Parra R.

Hospital Clínico Pontifica Universidad Católica de Chile.

Fono: 56-2-3543486

Correo Electrónico: rparra@med.puc.cl 


\section{Introducción}

En los últimos años el pronóstico de las cardiopatías congénitas ha mejorado significativamente. Parte del mayor éxito del manejo se debe al advenimiento de nuevas técnicas de imágenes no invasivas que han permitido mejorar la precisión diagnóstica anatómica y funcional, ayudando al planeamiento prequirúrgico y control postoperatorio. Es así, como la resonancia magnética (RM) cardíaca se ha consolidado como una herramienta de gran utilidad en el estudio de las cardiopatías ya que provee información necesaria y útil para una mejor evaluación y control de estos pacientes.

Es importante resaltar las ventajas de esta técnica, como ser un método no invasivo, no utilizar radiación ionizante, sumado a la excelente resolución de contraste para el estudio morfológico, vital en las cardiopatías congénitas ${ }^{1}$. La RM cardíaca permite además obtener información funcional y de dinámicas de flujo.

El objetivo de este trabajo es describir nuestra experiencia en la utilización de la RM cardíaca para la evaluación de las cardiopatías congénitas, los hallazgos más comunes y sus principales indicaciones.

\section{Método}

Se realizó un análisis retrospectivo de las RM cardíacas efectuadas a pacientes con cardiopatías congénitas entre los años 2002 y 2008, en el Hospital Clínico de la Pontifica Universidad Católica de Chile. Los exámenes fueron realizados en resonadores de 1.5 Tesla marca Philips (modelos Intera y Achieva) y Siemens (Modelo Magnetom Avanto). En el período inicial se realizó sólo estudio anatómico y desde mayo de 2003 estuvo disponible en nuestra institución el software necesario para realizar estudios funcionales y de dinámicas de flujo, esto último mediante mediciones por contraste de fase. En todos los exámenes estuvo presente el radiólogo para programar el protocolo de estudio de acuerdo al diagnóstico de derivación, en general en concordancia con lo reportado en cada patología, con lo recomendado por nuestros centros de referencia y en base también a la experiencia adquirida. Para todos fue indispensable contar con el diagnóstico de derivación e idealmente información específica requerida por el clínico. En aquellos pacientes en que no se señalaba el diagnóstico o la información en la orden no era suficiente, hubo comunicación directa con el tratante.

Una vez adquiridas las imágenes, se efectuó análisis en estación de trabajo para interpretación de los hallazgos anatómicos, obtención de curvas de flujo y reproceso de las imágenes angiográficas. En todos los pacientes se realizó como protocolo básico estudio anatómico completo con cortes estándar en secuencias de cine (sangre blanca): cortes axiales, cortes en eje largo de ambos ventrículos, eje corto, tractos de salida, cuatro cámaras, y estudio de función ventricular. En algunos casos se utilizó cortes estáticos ponderados en T1 (sangre negra).

Además, dependiendo del diagnóstico de derivación se realizaron cortes en los ejes que fuera necesario para mejor definición anatómica y/o relaciones anatómicas de las estructuras, o bien secuencias específicas para caracterización tisular en los casos de tumores o de sospecha de Displasia Arritmogénica del Ventrículo Derecho.

Desde el momento en que fue posible efectuar mediciones de flujo por contraste de fase, en todos los pacientes las mediciones de rutina incluyeron: aorta proximal, tronco de la arteria pulmonar, válvulas atrioventriculares y de acuerdo al diagnóstico se programaron mediciones adicionales, ya sea para estimar gradientes, medición del flujo pulmonar diferencial, graduación de estenosis de venas pulmonares, estimación de QP/QS, etc.

Además se utilizó estudio angiográfico y/o estudio de viabilidad miocárdica con medio de contraste paramagnético intravenoso, o marcación miocárdica (tagging) en los casos necesarios, según su patología.

En la revisión se incluyeron tanto pacientes en edad pediátrica como adultos. En los pacientes 
pediátricos menores de 5 años se utilizó sedación o anestesia. Los pacientes entre 6 y 9 años, sobre todo los más cercanos al rango superior de edad de este grupo y algunos mayores con patología asociada (como síndrome de Down) fueron evaluados caso a caso y en algunos de ellos fue posible realizar el estudio sin sedación ni anestesia, utilizando técnicas para adquirir las secuencias en respiración espontánea.

\section{Resultados}

Se efectuaron 180 resonancias magnéticas cardíacas en el período evaluado, en un total de 173 pacientes (en seis de ellos se efectuaron dos y en un paciente tres exámenes en el período de estudio). En cuanto al número de exámenes realizado, destaca un significativo y progresivo incremento principalmente en los últimos tres años (Fig. 1)

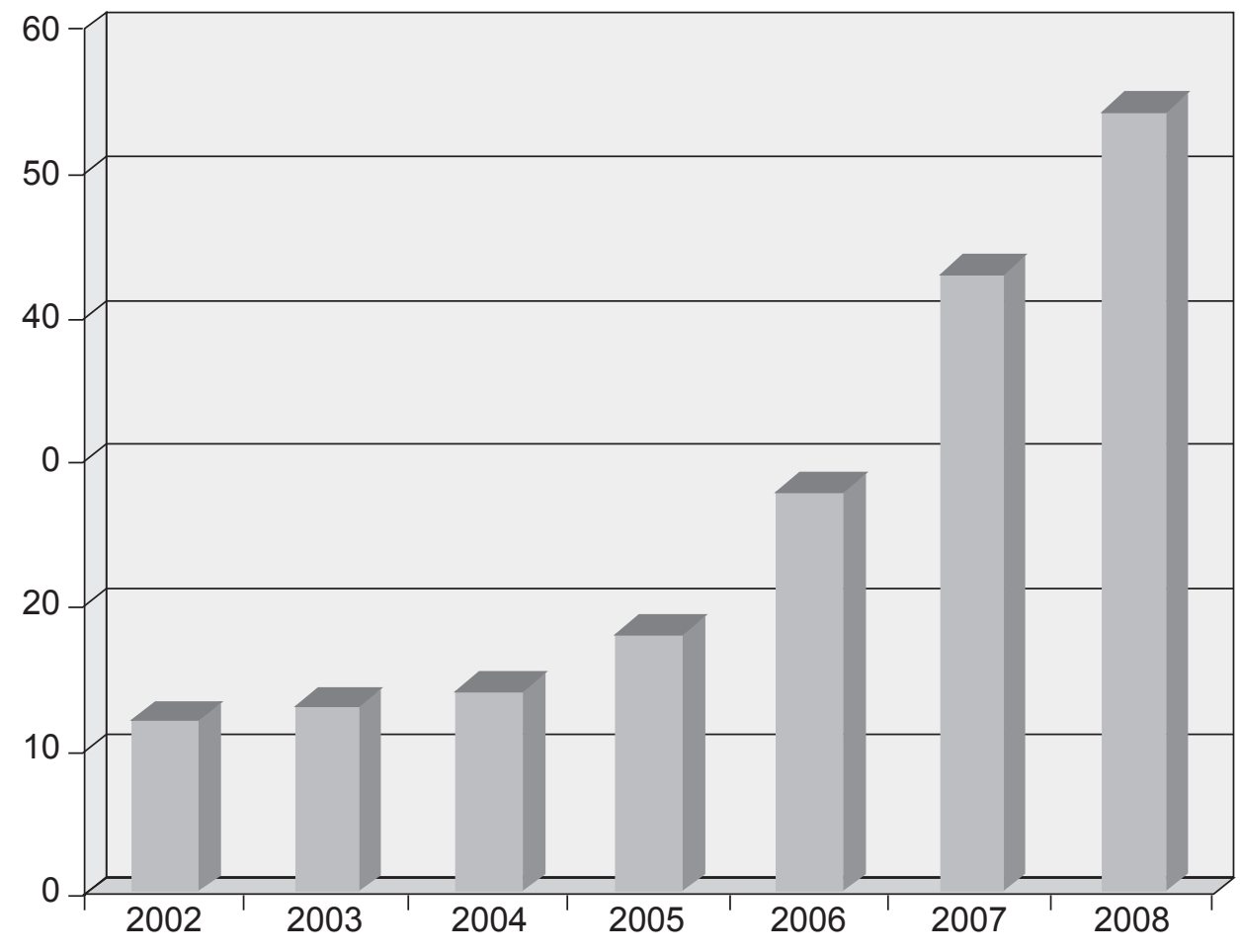

RM cardiac realizadas

Figura 1: Número de exámenes (Resonancia Magnética Cardíaca) en los últimos años.

La distribución por sexo fue 109 (63\%) pacientes masculinos y $64(37 \%)$ de sexo femenino.

Las edades oscilaron entre 1 día de vida hasta 69 años. Se crearon grupos de edad de la siguiente manera: Menores de 1 año = 35 pacientes; 2 a 10 años $=44$ pacientes; 11 a 20 años $=78$ pacientes; 21 a 40 años $=14$ pacientes y mayores de 41 años $=2$ pacientes, con lo que se puede observar que el grupo etario más común fue entre los 11 y 20 años de edad. Cabe destacar que de los pacientes menores de un año, 5 fueron recién nacidos, uno de ellos pre término, por lo que no hubo limitación en cuanto a edad y en especial en cuanto al peso para la realización del estudio. 
La principal indicación del estudio fue control postoperatorio, ya que la mayoría de los pacientes eran postquirúrgicos de Tetralogía de Fallot y el objetivo del examen fue evaluación del grado de insuficiencia pulmonar (fig 2), también la evaluación postquirúrgica del ventrículo único y transposición de grandes vasos. Otras indicaciones fueron evaluación prequirúrgica de diagnósticos establecidos por ecocardiografía, que requerían mayor detalle anatómico y de función, como en los pacientes con drenaje venoso anómalo con o sin estenosis (fig 3), coartación de aorta (fig 4), anomalía de Ebstein, entre otros. En tercer lugar las RM cardiacas fueron para evaluación diagnóstica inicial.
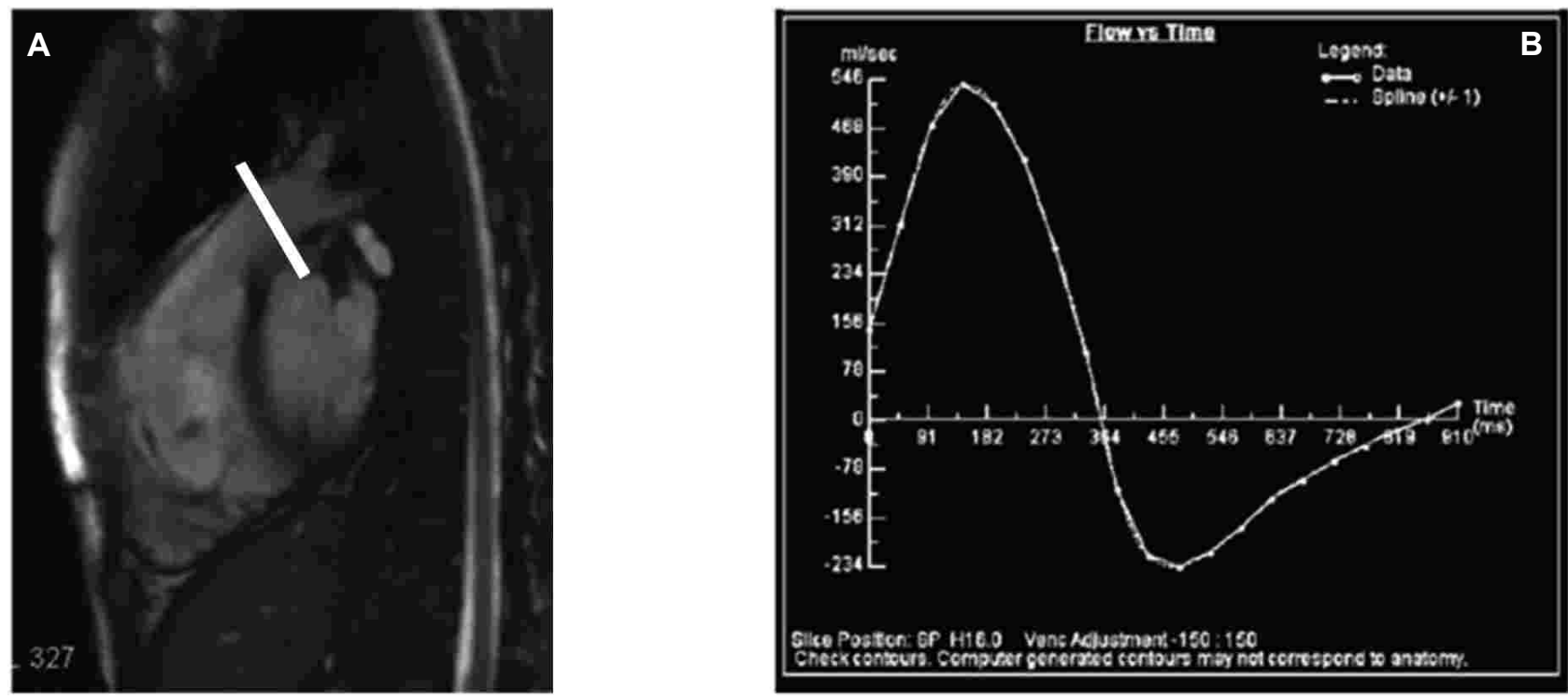

Figura 2: Tetralogía de Fallot operada. (a) Imagen del tronco de la arteria pulmonar que muestra el punto en que se efectúa la medición de flujo (línea roja). (b) Curva de flujo obtenida que muestra flujo anterógrado en sístole (positivo) y reverso en diástole (negativo), lo cual permite calcular la fracción de regurgitación pulmonar.
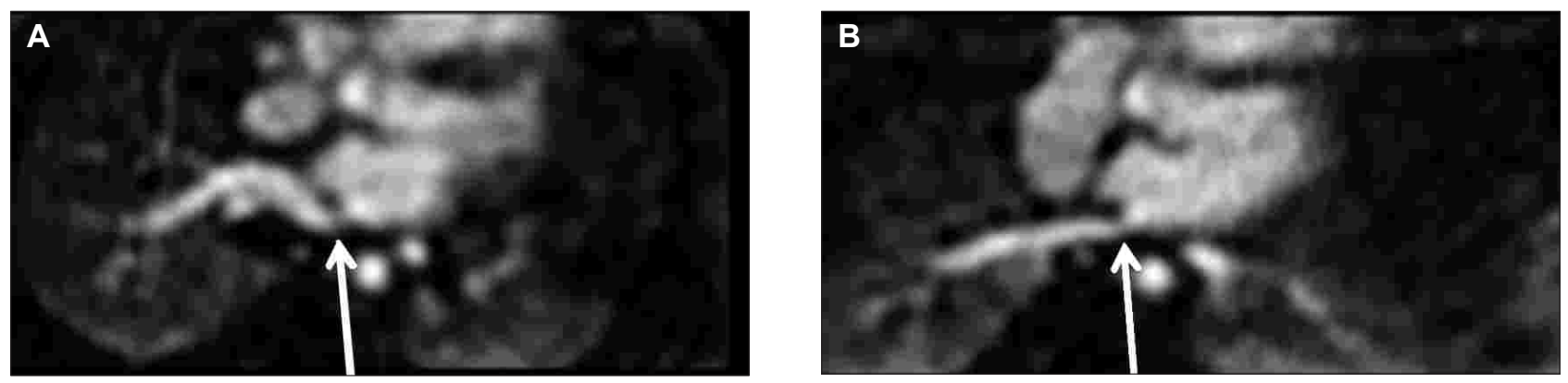

Figura 3: Paciente de 3 meses. Drenaje venoso anómalo pulmonar total operado. Reconstrucciones de Angio RM que muestran estenosis en la desembocadura de la vena pulmonar superior (a) e inferior (b) derecha. 

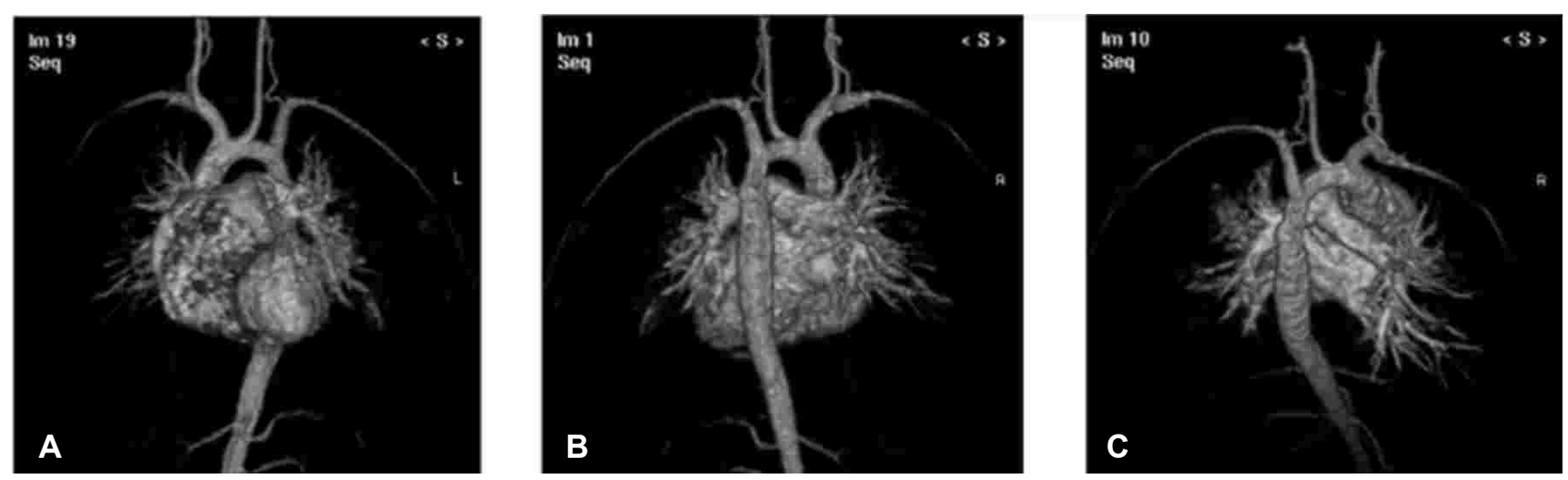

Figura 4: Control plastía de coartación aórtica. Reconstrucciones tridimensionales de Angio RM en vistas anterior (a) y posterior (b) y superior (c) que muestran discreta irregularidad en sitio de angioplastía, sin evidencias de estenosis significativa.

Adicionalmente a 39 pacientes con tetralogía de Fallot se les realizó viabilidad miocárdica. En dos de ellos se encontró zonas de fibrosis en la región del tracto de salida del ventrículo derecho y en uno extensas áreas de isquemia antero septal.

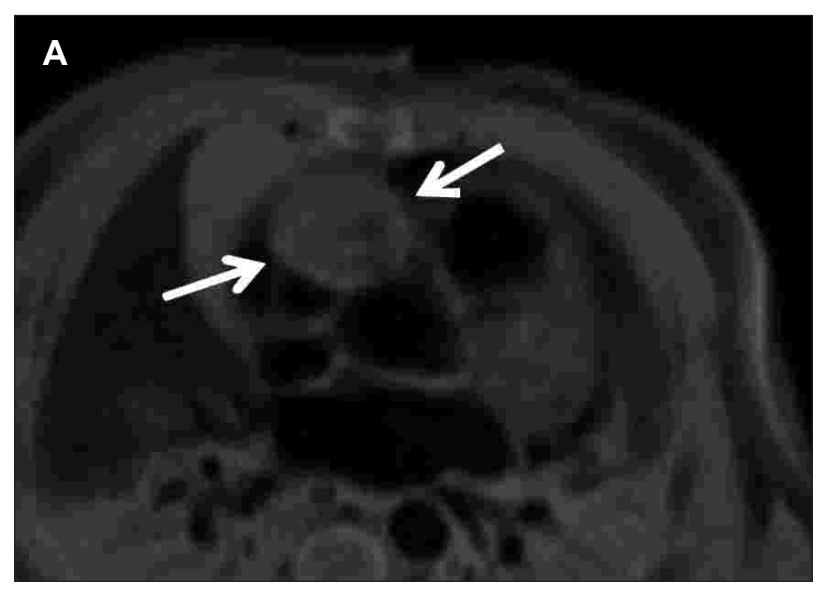

Otros hallazgos fueron masas auriculares (fig 5), aneurisma de orejuela izquierda (fig 6), miocardiopatía no compactada (fig 7), ventrículo único funcional, miocardiopatía dilatada, coartación de aorta, entre otros (Tabla 1).

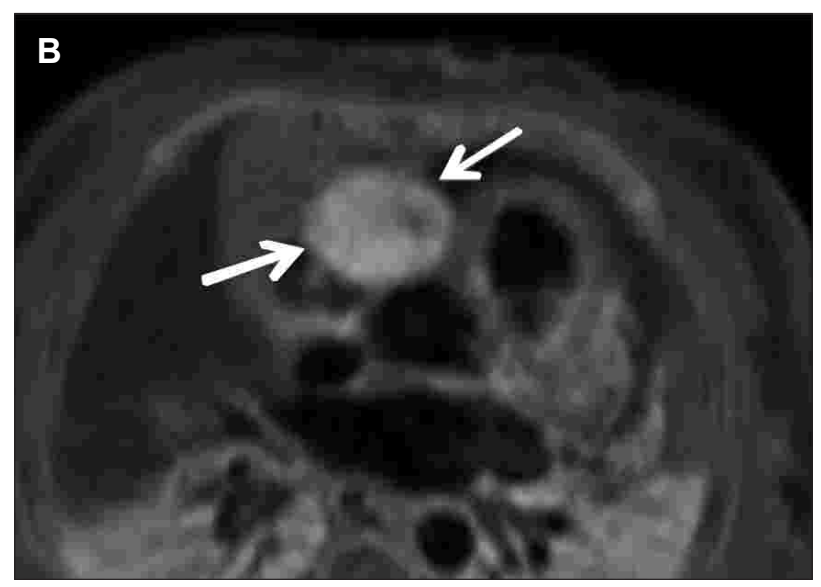

Figura 5: Cortes axiales ponderados en T1 con saturación grasa antes (a) y posterior (b) a la inyección de gadolinio endovenoso que muestran una masa de aspecto benigno en la aurícula derecha que se refuerza intensamente con el contraste paramagnético, compatible con un hemangioma (flechas) 
R. Parra , L. Cadavid, C. García, F. de Barbieri.
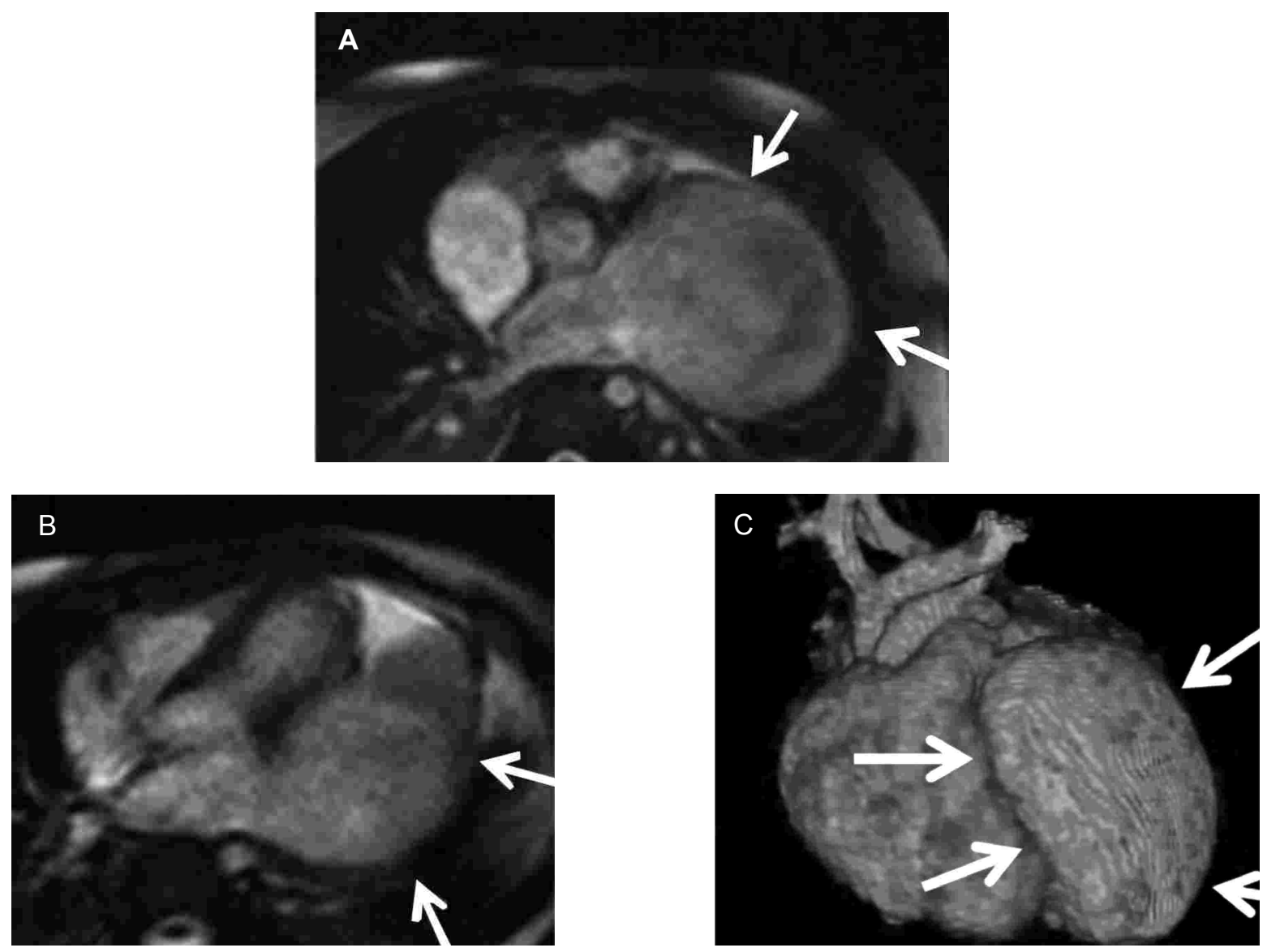

Figura 6: Cortes axial (a), en cuatro cámaras (b) y reconstrucción tridimensional de Angio RM en vista antero lateral izquierda(c) que muestran aneurisma gigante de la orejuela izquierda (flechas).
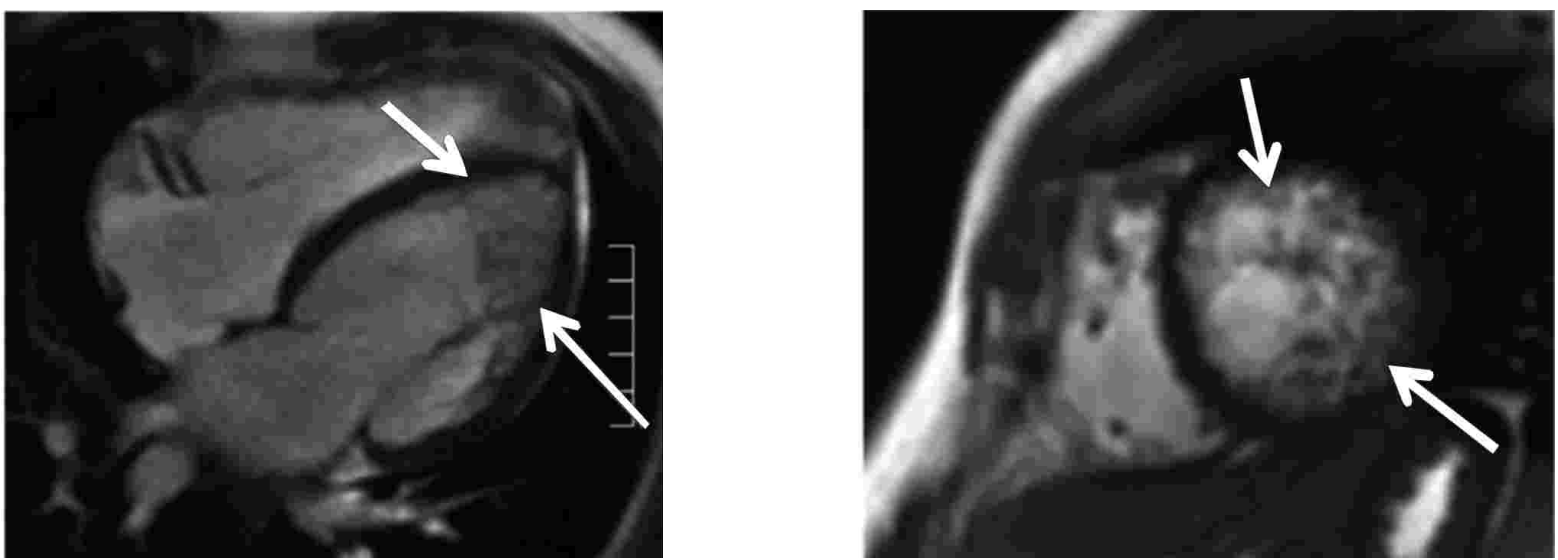

Figura 7: Miocardiopatía no compactada. Cortes en eje cuatro cámaras (a) y en eje corto (b) obtenidas de secuencias de cine que muestran la acentuación de trabeculación del VI (flechas), mayor en la región apical, propia de la Miocardiopatía no compactada. 
Tabla 1: Hallazgos por RM cardíaca

\begin{tabular}{lc}
\hline Hallazgos & No. Pacientes \\
\hline Tetralogía de Fallot & 55 \\
(viabilidad positiva en 2 pacientes y cayado aórtico derecho en 6 pacientes) & 18 \\
Drenaje venoso anómalo total o parcial pre y post quirúrgico, con o sin estenosis & 17 \\
Miocardiopatía no compactada & 10 \\
Ventrículo único & 9 \\
Miocardiopatía dilatada & 6 \\
Coartación de aorta & 6 \\
Shunt (CIA, CIV, ductus) & 6 \\
Anillos vasculares con compresión de la vía aérea, arteria subclavia derecha aberrante. & 6 \\
Control postoperatorio de transposición de grandes vasos & 7 \\
Tumores & 6 \\
Benigno (4) & 6 \\
Control tumor maligno (2) & \\
Síndromes de heterotaxia, & 4 \\
Anomalía de Ebstein & 4 \\
Agenesia de los velos pulmonares & 4 \\
Displasia arritmogénica & 3 \\
Otros: & \\
Aneurisma múltiples de la aorta , aneurisma del tronco de salida del ventrículo & \\
derecho (Fig. 15), Pentalogía de Cantrell, Cor triatriatum atípico, aneurisma de la & \\
aurícula izquierda, origen de la carótida izquierda desde la arteria pulmonar & 7 \\
Normal & 10 \\
\hline
\end{tabular}

Hubo 10 RM sin hallazgos de significado patológico cuya principal indicación fue la búsqueda de Displasia Arritmogénica de Ventrículo Derecho (Fig 8).

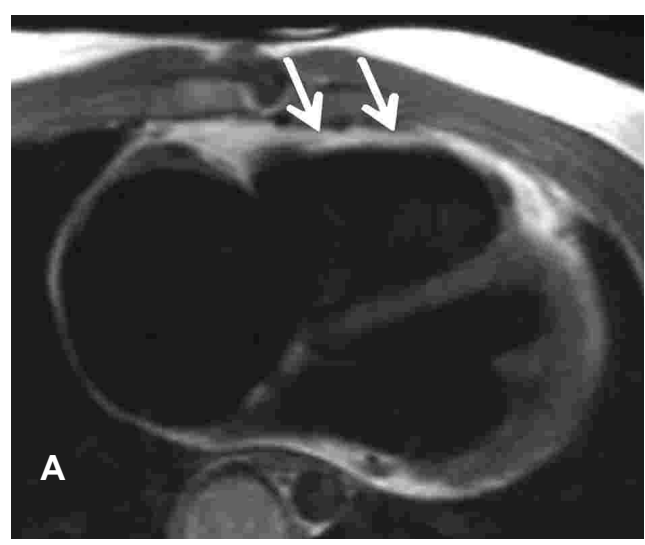

Durante estos 6 años, no se presentó ninguna complicación durante ni posterior al examen, ni reacción adversa con el uso del contraste endovenoso.

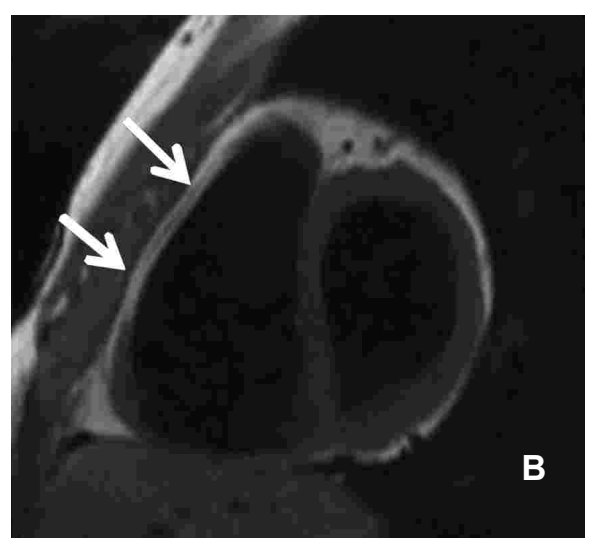

Figura 8: Displasia arritmogénica del VD. Cortes axial (a) y en eje corto (b) que muestran áreas de reemplazo adiposo en el miocardio del VD (flechas), lo que constituye un criterio diagnóstico mayor. 


\section{Discusión}

En los últimos 5 años, se ha hecho cada vez más evidente un cambio en los algoritmos para la evaluación de las cardiopatías congénitas. Es así como la Resonancia Magnética cardíaca se ha constituido como un excelente método diagnóstico dadas sus características ya ampliamente reportadas, y más aún con la aparición de técnicas actualmente en fase de difusión, como la adquisición isotrópica tridimensional que permite hacer reconstrucción de datos para el análisis anatómico con una visualización completa de las anomalías congénitas, tanto intra como extra cardíacas o con el perfeccionamiento de las técnicas ya existentes por ejemplo con la mejoría progresiva en la resolución temporal. 1-3

Otra de las ventajas de este método es el estudio funcional y de dinámicas de flujo sin utilización de radiación ionizante. Permite además obtener imágenes con excelente resolución espacial y de contraste, algunas veces sin utilización de medio de contraste. Por otro lado permite efectuar estudios de caracterización tisular, de Angio-Resonancia, de perfusión y viabilidad miocárdica 1,4.

Sin embargo, sus principales desventajas son la duración de los estudios (que en general varía entre 30 a 60 minutos), lo que hace necesario utilización de sedación o anestesia general principalmente en los pacientes menores de 8 años, para, especialmente en el segundo caso, mediante el control de la vía aérea obtener apneas y con ello una mejor resolución espacial y disminuir así los artefactos por movimiento. Por otro lado, el análisis de los datos en la estación de trabajo y el análisis de los valores y curvas obtenidas, requiere tiempo y dedicación de un profesional adecuadamente entrenado.

No obstante lo anterior, sigue siendo un método ideal dado que nos entrega información anatómica tanto intra como extra cardíaca, información de volumen / función ventricular sin asumir geometría; con medición de fracción de eyección, masa ventricular, caracterización del miocardio etc.; y de dinámicas de flujo en un solo estudio no invasivo que además no utiliza radiación ionizante, este último punto crucial, especialmente en pacientes pediátricos que requerirán múltiples exámenes durante su vida. ${ }^{1}$

Nuestro estudio descriptivo es un reflejo del avance de los métodos por imágenes y de la importancia de la RM para la evaluación de las cardiopatías. Es así como la curva ha ido en aumento. En el año 2002 se realizaron 12 resonancias y hasta octubre del año 2008 ya se habían superado ampliamente los años anteriores con 54 estudios realizados. Este dato soporta la evidencia de la importancia de la RM como una herramienta que proporciona datos útiles y necesarios tanto para el diagnostico y seguimiento pre y postoperatorio de la enfermedad cardiaca congénita.

Es así como por ejemplo en el control post operatorio de Tetralogía de Fallot, que fue la indicación más frecuente, permitió evaluar en forma precisa la fracción de regurgitación pulmonar, parámetros de volumen y función ventricular, en algunos casos comprobó la presencia de insuficiencia tricuspídea y en algunos casos fue compatible con disfunción diastólica del ventrículo derecho, todos parámetros que permitieron decidir la instalación o no de válvula protésica en situación pulmonar, en base a las indicaciones reportadas 5 con el fin de evitar el deterioro irreversible de la función del ventrículo derecho y disminuir la morbi-mortalidad post quirúrgica debido por ejemplo a la aumento de la probabilidad de muerte súbita en relación a la mayor dilatación ventricular1,5,6. Destacamos que a 39 pacientes con tetralogía de Fallot les realizamos estudio de viabilidad miocárdica. En dosde ellos encontramos zonas de fibrosis en la región del tracto de salida del ventrículo derecho y en uno extensas áreas de isquemia antero septal. Este hallazgo es importante dado que en la literatura aún no hay evidencia suficiente sobre la importancia de realizar viabilidad miocárdica en pacientes con cardiopatías congénitas y por lo tanto su utilidad, al igual que los estudios de perfusión miocárdica está aún por definir. 1,2

Otra de las patologías detectadas en nuestro estudio, es la miocardiopatía no compactada, 
Experiencia de 6 años en la evaluación de las cardiopatías congénitas con resonancia magnética.

entidad catalogada sólo recientemente dentro del grupo de las miocardiopatías y que corresponde a una alteración idiopática de origen congénito caracterizada por alteración de la estructura del miocardio del ventrículo izquierdo, condicionada por la detención del proceso de compactación normal.7

La RM cardíaca es ideal para la pesquisa de la displasia arritmogénica del ventrículo derecho, al igual que en la evaluación de masas cardíacas por su cualidad de permitir caracterización tisular, característica que en el caso de la displasia arritmogénica permite detectar el reemplazo adiposo del miocardio del ventrículo derecho además del volumen de éste y/o de áreas de diskinesia. Es también útil en el diagnóstico y seguimiento de pacientes con antecedente de coartación aortica; estimación de la magnitud del shunt, mediante la medición de la relación QP/QS en casos de comunicaciones interventriculares (CIV), auriculares (CIA), casos con colaterales aorto-pulmonares, etc. Permite también estimación de gradientes en casos de estenosis de vasos sanguíneos, medición precisa del flujo pulmonar diferencial. etc. 8

Es importante también resaltar la utilidad de la $\mathrm{RM}$ en pacientes recién nacidos o pacientes pequeños con cardiopatía compleja, en los que la RM puede ayudar a las decisiones quirúrgicas.
Por ejemplo en aquellos casos de hipoplasia ventricular límite, la RM nos permitirá determinar en forma precisa los volúmenes ventriculares para tomar adecuada decisión entre la corrección uni o biventricular. También en este grupo de edad, en los casos de drenaje venoso anómalo pulmonar permite, aparte de caracterizar la anatomía de los vasos, evaluar adecuadamente la repercusión de esta anomalía, tanto con la medición de la magnitud del shunt (QP/QS), como en la repercusión en el volumen y función de las cavidades, permitiendo así adecuada decisión en cuanto a la necesidad o no y a la oportunidad de la corrección quirúrgica. Por otro lado en el control post operatorio de los pacientes con drenaje venosos anómalo fue de mucha utilidad para estimar el pronóstico, al permitir caracterizar la anatomía de las estenosis u obstrucción de las venas pulmonares, así como la repercusión tanto en las curvas de flujo de las venas, como la repercusión en el flujo arterial pulmonar y de esta forma, en la mayoría de los casos permitió decidir si era posible o no ofrecer un nuevo tratamiento. 9

En conclusión la RM cardíaca es un método no invasivo que revela el detalle anatómico, funcional y de dinámicas de flujo, toda información importante para el planeamiento quirúrgico, seguimiento y control de las cardiopatías congénitas.

\section{Referencias}

1. NORTON KI, TONG C, GLASS RB, NIELSEN JC. Cardiac MR Imaging Assessment Following Tetralogy of Fallot Repair. Radiographics 2006; 26:197-211.

2. TAYLOR AM. Cardiac imaging: MR or CT? Which to use when. Pediatr Radiol 2008; 38: S433-S438.

3. HIGGINS CH. Cardiac Imaging. Radiology 2000; 217: 4-10

4. SENA L. Cardiac MR imaging: from physics to protocols. Pediatr Radiol 2008; 38: S185-S191.

5. GEVA T, AYRES NA, PAC FA, PIGNATELLI R. Quantitative Morphometric Analysis of Progressive Infundibular Obstruction in Tetralogy of Fallot. Circulation. 1995; 92: 886-892.

6. GROTHOFF M, SPORS B, ABDUL-KHALIQ H, GUTBERLET $M$. Evaluation of postoperative pulmonary regurgitation after surgical repair of tetralogy of Fallot: comparison between Doppler echocardiography and MR velocity mapping. Pediatr Radiol 2008; 38: 186-191.

7. STÖLLBERGER C, FINSTERER J. Evident, but poorly defined: left ventricular hypertrabeculation/noncompaction and its diagnostic criteria: reply. Eur. Heart J 2005; 26: 1144-1145.

8. KRISHNAMURTHY R. Pediatric cardiac MRI: anatomy and function. Pediatr Radiol 2008; 38: S192-S199

9. GROSSE-WORTMANN L, AL-OTAY A, GOO HW, MACGOWAN CK, COLES JG, BENSON LN, et al. .Anatomical and Functional Evaluation of Pulmonary Veins in Children by Magnetic Resonance Imaging. J Am Coll Cardiol 2007; 49: 993-1002. 